\title{
A very simple and chemoselective air oxidation of benzoins to benzils using alumina
}

\author{
Konstantinos Skobridis, ${ }^{\mathrm{a}}$ Vassiliki Theodorou, ${ }^{\mathrm{a}}$ and Edwin Weber*b \\ ${ }^{a}$ Department of Chemistry, University of Ioannina, GR-451 10 Ioannina, Greece \\ ${ }^{b}$ Institut für Organische Chemie, TU Bergakademie Freiberg, Leipziger Straße 29, \\ D-09596 Freiberg/Sachs., Germany \\ E-mail: kskobrid@,cc.uoi.gr
}

\begin{abstract}
An efficient and facile air oxidation of benzoins in the presence of the heterogeneous catalyst aluminum oxide, avoiding the use of noxious metal compounds as oxidizing agents has been developed. It thus constitutes a very simple, environmentally friendly, clean, economical and selective method for the aerobic preparation of benzils from the corresponding benzoins.
\end{abstract}

Keywords: Benzoins, benzils, air oxidation, alumina, chemoselectivity

\section{Introduction}

Benzils or $\alpha$-dicarbonyl compounds, in general, are important materials due to their practical applications, i.e. as starting materials for the synthesis of heterocycles ${ }^{1}$ and as photosensitive agents. $^{2}$ The oxidation of benzoins has been accomplished by several oxidative reagents such as nitric acid, thallium nitrate, copper sulfate, copper acetate, ammonium nitrate, bismuth oxide and bismuth nitrate, iron (II) thiolate and oxone, trichlorooxyvanadium and vanadium oxide, titanium (IV) chloride and triethylamine ${ }^{3}$ or by molecular oxygen using microwave irradiation on zeolite A or clayfen, ${ }^{4}$ or by nickel hydrotalcite activation. ${ }^{5}$ However, while all these methods can generate the desired products efficiently, many of the above oxidants are toxic and hazardous metal compounds, generating waste disposal problems. An indirect electrochemical method was, also, recently used for the oxidative transformation of benzoins to benzils. ${ }^{6}$ The cleanest and the most ideal reagent for oxidation reactions would be air. Thus, oxidative transformations of organic compounds effected by air oxygen are attractive from an economic and environmental point of view, and have received much attention in recent years. ${ }^{7}$ On the other hand, catalytic processes induced by heterogeneous catalysts ${ }^{8}$ have a distinct advantage in view of ease of handling, simple work-up and regenerability. 
Herein, we describe such an extremely simple and efficient transformation process which is the chemoselective aerobic oxidation of benzoins to benzils, supported by commercially available aluminum oxide as a solid catalyst, without the use of any metal salts as oxidizing agents or other reagents (Scheme 1). The oxidation is performed heterogeneously within the micropores of the solid alumina.

The role of alumina as a heterogeneous catalyst on several organic reactions, such as oxidation, reduction and displacement reactions, is known from the previous work of Posner. ${ }^{9}$ These reactions are heterogeneous, taking place at the alumina surfaces.

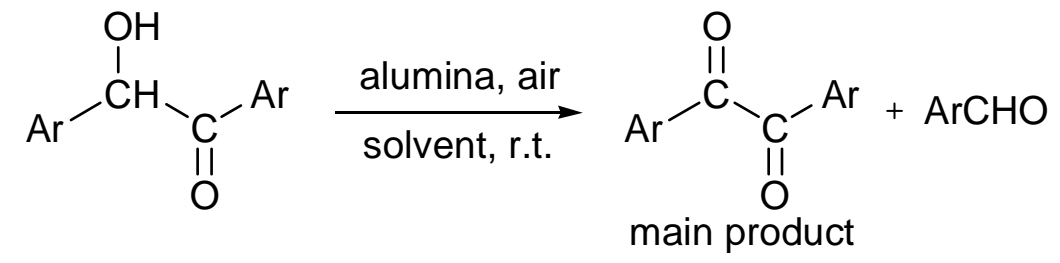

Aryl = phenyl, 4-anisyl, 2-furanyl, 4-tolyl, 4-chlorophenyl

Scheme 1. Oxidation reaction of aryloins with air oxygen in the presence of alumina.

\section{Results and Discussion}

In order to study the optimization of the reaction conditions and to evaluate the parameters that may affect the reaction, we carried out the oxidation experiments under an air atmosphere using acidic, neutral and basic alumina, i.e. $\mathrm{pH} 4.5,7.0$ and 9.5, respectively. The reaction rate was found to be strongly influenced by the $\mathrm{pH}$ of the alumina, and the best result was obtained with basic aluminum oxide $(\mathrm{pH}$ 9.5). The higher reaction rates with basic aluminum oxide may be attributed to the easier abstraction of an $\alpha$-proton by a basic site of the catalyst, ${ }^{10}$ leading to a stabilized enediolate anion which reacts further (Figure 1). The reaction rate was also influenced significantly by the amount of alumina, being retarded with the decrease of it. In a representative procedure (Table 1), $1 \mathrm{mmol}$ of benzoin when treated with $5 \mathrm{~g}$ of neutral alumina in $20 \mathrm{ml}$ of $\mathrm{CH}_{2} \mathrm{CI}_{2}$ required $55 \mathrm{~min}$ to be transformed to benzil, while with $3 \mathrm{~g}$ and $2 \mathrm{~g}$ of alumina the appropriate time became $3 \mathrm{~h}$ and $7 \mathrm{~h}$, respectively. Alternatively, with $5 \mathrm{~g}, 3 \mathrm{~g}$, and $2 \mathrm{~g}$ of basic alumina the appropriate time was $35 \mathrm{~min}, 2 \mathrm{~h}$ and $4 \mathrm{~h}$, respectively.<smiles>O=C(O)C(O)Br</smiles>

Figure 1. Suggested anionic intermediate during catalytic benzoin oxidation. 
Furthermore, in order to determine the solvent effect on the reaction rate, we tried the reaction in apolar, polar aprotic and protic solvents, such as toluene, dichloromethane, acetonitrile, and methanol. The conclusion is that the reaction rate increases in solvents with decreasing polarity (Table 1). Among the studied solvents, the less polar toluene and dichloromethane were found to be the best solvents for this reaction protocol. Apolar solvents, such as hexane and carbon tetrachloride, were not tried due to the low solubility of benzoins in these solvents. Acetonitrile, as a more polar aprotic solvent, can also be used but with a lower reaction rate. On the other hand, the protic solvent methanol obviously retards the oxidation of benzoin. Probably, this is due to the stabilization of benzoin through hydrogen bonding with methanol.

Table 1. Aerobic oxidation of benzoins to benzils with alumina ${ }^{a}$

\begin{tabular}{cccccc}
\hline & & \multicolumn{3}{c}{ Time of aryloin complete reaction $^{\mathrm{b}}$} & Yield \\
Aryloin & Solvent & $\mathrm{pH} \mathrm{4,5}$ & $\mathrm{pH} \mathrm{7,0}$ & $\mathrm{pH} \mathrm{9,5}$ & $(\%)^{\mathrm{c}}$ \\
\hline benzoin & $\mathrm{MeOH}$ & $\sim 100 \mathrm{~h}$ & $11 \mathrm{~h}$ & $7 \mathrm{~h} \mathrm{30min}$ & 85 \\
benzoin & $\mathrm{CH}_{3} \mathrm{CN}$ & $4 \mathrm{~h} \mathrm{30min}$ & $1 \mathrm{~h} \mathrm{30min}$ & $40 \mathrm{~min}$ & 76 \\
benzoin & $\mathrm{C}_{6} \mathrm{H}_{5} \mathrm{CH}_{3}$ & $1 \mathrm{~h} \mathrm{30min}$ & $45 \mathrm{~min}$ & $30 \mathrm{~min}$ & 65 \\
benzoin & $\mathrm{CH}_{2} \mathrm{Cl}_{2}$ & $2 \mathrm{~h} \mathrm{30min}$ & $55 \mathrm{~min}$ & $35 \mathrm{~min}$ & 67 \\
anisoin & $\mathrm{CH}_{2} \mathrm{Cl}_{2}$ & $10 \mathrm{~h}$ & $3 \mathrm{~h}$ & $1 \mathrm{~h} 10 \mathrm{~min}$ & 69 \\
furoin & $\mathrm{CH}_{2} \mathrm{Cl}_{2}$ & $25 \mathrm{~min}$ & $15 \mathrm{~min}$ & $10 \mathrm{~min}$ & 82 \\
4,4'-dimethylbenzoin & $\mathrm{CH}_{2} \mathrm{Cl}_{2}$ & $5 \mathrm{~h} \mathrm{30min}$ & $1 \mathrm{~h} \mathrm{35min}$ & $1 \mathrm{~h}$ & 55 \\
4,4'-dichlorobenzoin & $\mathrm{CH}_{2} \mathrm{Cl}_{2}$ & $2 \mathrm{~h} 15 \mathrm{~min}$ & $35 \mathrm{~min}$ & $14 \mathrm{~min}$ & 58 \\
\hline
\end{tabular}

a $1 \mathrm{mmol}$ aryloin, $5 \mathrm{~g}$ alumina, $20 \mathrm{ml}$ solvent, room temperature, atmospheric pressure and open glass containers.

${ }^{\mathrm{b}}$ The reaction time was determined by monitoring the vanishing spot of the starting aryloin on TLC.

${ }^{c}$ Yields refer to chromatographically isolated pure compounds (column, silica, $\mathrm{CH}_{2} \mathrm{Cl}_{2}$ as eluent, neutral alumina. With acidic and basic alumina the yields were comparable).

In order to evaluate the scope of this protocol, we also studied the air oxidation of anisoin and furoin in dichloromethane (Table 1). Compared to the benzoin, the oxidation of anisoin takes place much more slowly. This could be attributed to a stabilizing effect of the enediolate anion by the electron donating 4-methoxy groups. Probably the methoxy group by its $+\mathrm{M}$ effect retards the abstraction of the proton in the first step of the suggested mechanism, slowling down the reaction rate. The same effect, but in a lower degree, was observed with 4,4'-dimethylbenzoin, due to the $+\mathrm{I}$ effect of the methyl groups. The reverse is the case of the oxidations for 4,4'dichlorobenzoin and furoin, which are very high, even in the presence of acidic alumina. This may be a result of the strong -I effect of the heterocyclic oxygen, close to the carbanion. In 
neutral alumina, benzil, anisil, furil, 4,4'-dimethylbenzil and 4,4'-dichlorobenzil were obtained in good yields, 55\%-85\% (Table 1). In all other conditions, the yields were comparable. Yet, some loss of yield occurred owing to partial decomposition of the aryloins to ArCHO, possibly due to a retro-reaction of the benzoin condensation. The recovered alumina can be reused up to three times since loss of activity was observed after that.

Although the exact mechanism of this novel alumina catalyzed aerobic oxidation reaction is not yet totally clear, all the above data suggest that the reaction proceeds through an anionic intermediate, which reacts further with molecular oxygen to give benzil. The more basic alumina, the less polar solvent and the more acidic benzoin constitute the best conditions for the reaction.

A noteworthy finding is that alcohols without a carbonyl group at the $\alpha$-position, i.e. benzylic alcohols, benzhydrols and cyclohexanol or aldehydes without neighboring hydroxy groups, i.e. geranyl acetaldehyde, anisaldehyde and vanillin, do not react under the reaction conditions, showing that the aryloin system is selective in the oxidation. It is also of interest to know that the oxidation does not proceed with other basic solid substrates, e.g. $\mathrm{K}_{2} \mathrm{CO}_{3}$, confirming the catalytic role of alumina. Finally, the reaction rate is not affected by the addition of the radical inhibitor 2-tert-butyl-4-hydroxyanisole, showing that free radical intermediates are not involved in the present oxidation course.

In summary, we have demonstrated that alumina can act as an efficient catalyst for the chemoselective air oxidation of benzoins to benzils through an environmentally acceptable process. The products, obtained in high yields, are isolated very easily and alumina catalyzed air oxidation, despite the inconvenience of using a large quantity of it, may serve as a highly competitive substitute for the recently described electrochemical oxidation method of benzoins to benzils, ${ }^{5}$ rendering superfluous electrochemical equipment. Further improvement of the parameters for the alumina catalyzed oxidation, to be applied to the synthesis of other products, is being under investigation in our laboratory, as well as exploring the mechanistic details of the oxidation process.

\section{Experimental Section}

General Procedures. The corresponding aryloin $(1.0 \mathrm{mmol})$ was added to a suspension of the respective aluminum oxide $(5 \mathrm{~g})$ in dichloromethane $(20 \mathrm{ml})$ as the suitable solvent (Table 1$)$. The resulting suspension was stirred vigorously at room temperature under air atmosphere, until the benzoin was no longer detectable by TLC analysis. The aluminum oxide was filtered off and washed successively with dichloromethane, methanol and dichloromethane. The solvent was evaporated, and hexane/diethylether $(5 \mathrm{ml})$ was added to remove the aldehyde. The resulting solid was filtered and dried. Further purification may be carried out by column chromatography $\left(\mathrm{SiO}_{2}, \mathrm{CH}_{2} \mathrm{Cl}_{2}\right.$ as eluent) to afford the products, benzils, as proven by melting points and ${ }^{1} \mathrm{H}$ NMR spectra, being identical to the authentic samples. ${ }^{12}$ The collected aluminum oxide was dried at $100{ }^{\circ} \mathrm{C}$ for $2 \mathrm{~h}$ and stored at r.t. overnight, in order to be reused. 
Materials. Alumina (Brockmann grade I; particle size: 0.05-0.15 mm; basic: $\mathrm{pH} 9.5 \pm 0.5$; neutral: $\mathrm{pH} 7.0 \pm 0.5$; acidic: $5.5 \pm 0.5$ ). The starting benzoins were purchased from Aldrich, except for 4,4'-dimethylbenzoin and 4,4'-dichlorobenzoin which were prepared according to the procedure described for the preparation of benzoin. ${ }^{11}$

\section{References}

1. (a) Vollhardt, K. P. C.; Schore, N. E. Organic Chemistry, $2^{\text {nd }}$ Edn., Freeman: New York, 1994, pp 924-929. (b) Gill, G. B. In Comprehensive Organic Synthesis Pattenden, G., Ed.; Pergamon: New York, 1991, Vol. 3, pp 821-838. (c) Kirihara, M.; Ochiai, Y.; Takizawa, S.; Takahata, H.; Nemoto, H. Chem. Commun. 1999, 1387. (d) Wynberg, H.; Kooreman, H. J. J. Am. Chem. Soc. 1965, 87, 1739. (e) Rothkopf, H. W.; Wöhrle, D.; Müller, R.; Kossmehl, G. Chem. Ber. 1975, 108, 875.

2. Matsushita Electric Industrial Co. Ltd., Jpn. Kokai Tokkyo Koho, 8198203, 1981; Chem. Abstr. 1981, 95, 188163v.

3. (a) Buck, J. S.; Jenkins, S. S. J. Am. Chem. Soc. 1929, 51, 2163. (b) McKillop, A.; Swann, B.; Ford, M. E.; Taylor, E. C. J. Am. Chem. Soc. 1973, 95, 3461. (c) Hartman, W. W.; Dickey, J. B. J. Am. Chem. Soc. 1933, 55, 1228. (d) Ruggli, P.; Zeller, P. Helv. Chim. Acta 1945, 28, 741. (e) Weiss, M.; Appel, M. J. Am. Chem. Soc. 1948, 70, 3666. (f) Righy, W. J. Chem. Soc. 1951, 793. (g) Tymonko, S. A.; Nattier, B. A.; Mohan, R. S. Tetrahedron Lett. 1999, 40, 7657. (h) Sun, W. Y.; Ueyama, N.; Nakamura, Tetrahedron, 1993, 49, 1357. (i) Varma, R.; Dahiya, R.; Kumar, D. Molecules 1998, 2, 82. (j) Kirihara, M.; Ochiai, Y.; Takizawa, S.; Takahata, H.; Nemoto, H. Chem. Commun. 1999, 1387. k) Velusamy, S.; Punniyamurthy, T. Org. Lett. 2004, 6, 217. (1) Periasamy, M. Arkivoc 2002, (vii) 151-156.

4. (a) Balalaie, S.; Golizeh, M.; Hashtroudi, M. S. Green Chemistry 2000, 2, 277. (b) Varma, R. S.; Dahiya, R. Tetrahedron Lett. 1997, 38, 2043.

5. Choudary, B. M.; Kantam, M. L.; Rahman, A.; Reddy, C. V.; Rao, K. K. Angew. Chem. 2001, 111, 763; Angew. Chem. Int. Ed. 2001, 113, 785.

6. Okimoto, M.; Takahashi, Y.; Nagata, Y.; Sasaki, G.; Numata, K. Synthesis 2005, 705.

7. Nishiyama, N.; Nakagawa, Y.; Mizuno, N. Angew. Chem. 2001, 113, 3751. Angew. Chem. Int. Ed. 2001, 40, 3639.

8. Lopez, J.; Valente, J. S.; Clacens, J. M.; Figueras, F. J. Catal. 2002, 208, 30.

9. (a) Posner,G. H.; Rogers, D. Z.; Kinzing, C. M.; Gurria, G. M. Tetrahedron Lett. 1975, 16, 3597. (b) Posner,G. H.; Runquist, A.W. Tetrahedron Lett. 1975, 16, 3601. (c) Posner,G. H.; Perfetti, R. B.; Runquist, A.W. Tetrahedron Lett. 1976, 17, 3499. (d) Posner,G. H.;

Chapdelaine, M. J. Tetrahedron Lett. 1977, 18, 3227.

10. Perri, J. B. J. Phys. Chem. 1965, 69, 220.

11. Vogel's Textbook of Practical Organic Chemistry, $5^{\text {th }}$ edn, $\mathrm{p} 1043$.

12. (a) Okimoto, M.; Itoh, T.; Chiba, T. J. Org. Chem. 1996, 61, 4835. (b) Zhang, G. S.; Shi, Q. Z.; Chem, M. F.; Cai, K. Synth. Commun. 1997, 27, 9534. 